\title{
Particular superintegrability of 3-body (modified) Newtonian Gravity
}

\author{
Alexander V. Turbine1* and Juan Carlos Lopez Vieyrdi \\ Instituto de Ciencias Nucleares, Universidad Nacional Autónoma de México, \\ Apartado Postal 70-543, 04510 México, D.F., Mexico
}

( $\Omega$ Dated: April 24, 2020)

\begin{abstract}
It is found explicitly 5 Liouville integrals in addition to total angular momentum which Poisson commute with Hamiltonian of 3-body Newtonian Gravity in $\mathbb{R}^{3}$ along the Remarkable Figure-8-shape trajectory discovered by Moore-Chenciner-Montgomery. It is checked that they become constants of motion along this trajectory. Hence, 3-body choreographic motion on Figure-8-shape trajectory in $\mathbb{R}^{3}$ Newtonian gravity (Moore, 1993), as well as in $\mathbb{R}^{2}$ modified Newtonian gravity by Fujiwara et al, 2003, is maximally superintegrable. It is conjectured that any 3-body potential theory which admit Figure-8-shape choreographic motion is superintegrable along the trajectory.
\end{abstract}

In 1993 C. Moore [1] discovered a new phenomenon in 3-body classical chaotic dynamics: three particles of equal masses in $\mathbb{R}^{3}$ Newtonian gravity can perform periodic motion on the same Figure-8-shape closed trajectory with zero total angular momentum and equal time delay without collisions. Later it was confirmed rigorously by Chenciner-Montgomery [2], who also proved that the trajectory is non-algebraic curve; it was also demonstrated its certain stability towards small perturbations of initial data [3]. This phenomenon was called choreography [4]. It was studied intensely since 2000 for different number of particles and various pairwise potentials being mostly limited to planar trajectories. It manifests the existence of moving equilibrium configuration: at least, for attractive pairwise potentials the motion can not be stopped due to a possibility of collapse otherwise, thus, a standard steady equilibrium configuration at zero velocities does not exist. The goal of the present Letter is to demonstrate that 3-body choreography on the Remarkable Figure Eight trajectory, as was called in [2], in $\mathbb{R}^{3}$ Newtonian Gravity is characterized by 7 constants of motion, which is the maximal possible amount, hence, being maximally superintegrable. Furthermore, there exist 6 independent functions on phase space which Poisson-commute with Hamiltonian along this trajectory. Hence, the Hamiltonian is maximally superintegrable along the Remarkable Figure Eight trajectory. As the first step we consider a $\mathbb{R}^{2}$ modified Newtonian gravity theory proposed by Fujiwara et al, [5], which allows the 3-body choreographic motion on algebraic lemniscate by Jacob Bernoulli (1694), and we construct explicitly seven, polynomial in coordinates and momenta, constants of motion along the trajectory.

We begin by introducing the notion of particular integral [6] in classical mechanics. Take a function $I(p, x)$ on classical phase space $(p, x)$, define the Hamiltonian $H(p, x)$ and calculate the Poisson bracket $\{H, I\}$. If for some trajectory(ies) in phase space the Poisson bracket vanishes, the function $I(p, x)$ is called a particular Liouville integral, it becomes constant (of motion) on the trajectory: the corresponding trajectory is called particular. Otherwise, if the Poisson bracket vanishes for any trajectory, $I(p, x)$ is a Liouville or global integral; it implies the r.h.s. of the Poisson bracket is zero identically. In order to have a non-trivial dynamics the maximal number of functionally independent integrals (including the Hamiltonian) should not exceed the dimension of phase space $d_{p h}=2 d_{c}$ minus one. When the number of integrals is bigger than the dimension of the coordinate space $d_{c}$, the dynamics is called superintegrable. It is known that in the case of maximal superintegrability where all $\left(d_{p h}-1\right)$ integrals are global, all bounded trajectories in coordinate space are closed (and periodic) [7], as for a concrete example see for instance [8]. It is natural to attempt to generalize the statement of [7]: even though some integrals are particular and emerging constants of motion occur for the same particular trajectory, the maximal particular superintegrability leads to closed particular trajectory. It will be shown that for 3-body Newtonian choreography and one on algebraic lemniscate it is the case. Note that following the well-known H. Poincare theorem the 3-body Newtonian dynamics in relative space is characterized by two global integrals, energy and total angular momentum, only.

(A) Modified $\mathbb{R}^{2}$ Newtonian gravity: the example of inverse problem. In 2003 Fujiwara et al, [5] solved the inverse problem: by taking the algebraic lemniscate on $(x, y)$-plane

$$
\left(x^{2}+y^{2}\right)^{2}=c^{2}\left(x^{2}-y^{2}\right),
$$

as the trajectory, where without loss of generality one can take $c=1$, it was shown that is a potential problem for 3 unit mass, point-like particles subject to pairwise potentials,

$$
V=\sum_{i<j}^{3}\left\{\frac{1}{4} \ln r_{i j}^{2}-\frac{\sqrt{3}}{24} r_{i j}^{2}\right\} \equiv \frac{1}{4} \ln I_{1}-\frac{\sqrt{3}}{24} I_{2},
$$

at zero angular momentum. Here

$$
r_{i j}=\sqrt{\left(\mathbf{x}_{i}-\mathbf{x}_{j}\right)^{2}},
$$

is relative distance between particles $i$ and $j$.

The first attractive terms in (2) represents 3-body $\mathbb{R}^{2}$ Newtonian potential at gravitational constant $G=1 / 2$, 


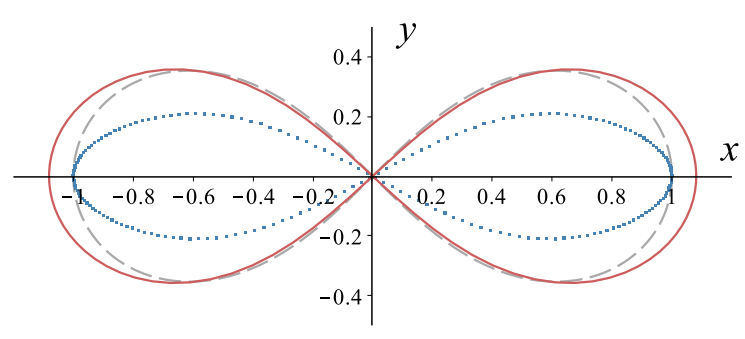

FIG. 1: Figure Eight trajectories for 3 unit mass particles: algebraic lemniscate - grey (dashed) line, $\mathbb{R}^{2}$ gravity -blue (dotted) line, $\mathbb{R}^{3}$ gravity - red (solid) line

while the second repulsive term is square of hyperradius in the space of relative motion. Each pairwise potential in (2) has maximum at $r_{i j}^{(\max )}=1.8612$. It can be shown that the motion on the trajectory is confined to the domain $r_{i j}^{(\min )} \leq r_{i j} \leq r_{i j}^{(\max )}$, where $r_{i j}^{(\min )}=0.6813$, thus, it never enters into the domain of configuration space, where the repulsion term $\sim I_{2}$ dominates.

It is known that the algebraic lemniscate (10) is parametrized by

$$
x(t)=c \frac{\operatorname{sn}(t, k)}{1+\operatorname{cn}^{2}(t, k)} \quad, \quad y(t)=c \frac{\operatorname{sn}(t, k) \operatorname{cn}(t, k)}{1+\operatorname{cn}^{2}(t, k)},
$$

see e.g. [5], where $\operatorname{sn}(t, k), \operatorname{cn}(t, k)$ are Jacobi elliptic functions, $k \in[0,1]$ is elliptic modulus, which defines via complete elliptic integral, the real period $T=$ $4 \int_{0}^{1} \frac{d x}{\sqrt{\left(1-x^{2}\right)\left(1-k^{2} x^{2}\right)}}: x(t)=x(t+T), y(t)=y(t+T)$. The evolution is defined by

$$
\begin{aligned}
x_{1}(t)=x(t) \quad, \quad y_{1}(t)=y(t), \\
x_{2}(t)=x(t+T / 3) \quad, \quad y_{2}(t)=y(t+T / 3), \\
x_{3}(t)=x(t-T / 3) \quad, \quad y_{3}(t)=y(t-T / 3),
\end{aligned}
$$

for the first, second and third particles, respectively. By straightforward calculation using Maple 18 in 20-digit arithmetic one can check that the center-of-mass is conserved

$$
\mathbf{X}_{\mathrm{CM}}(t)=\mathbf{x}_{1}+\mathbf{x}_{2}+\mathbf{x}_{3}=0
$$

and fixed

$$
\mathbf{V}_{\mathrm{CM}}(t)=\mathbf{v}_{1}+\mathbf{v}_{2}+\mathbf{v}_{3}=0,
$$

for elliptic modulus

$$
k_{0}^{2}=\frac{2+\sqrt{3}}{4}=\left(\frac{1+\sqrt{3}}{2 \sqrt{2}}\right)^{2} \simeq 0.933012701892 \ldots,
$$

only [5]. It corresponds to period $T=11.07225 \ldots$ There exist times along the evolution, given by $t=\frac{T}{2} n$, $n=0,1,2, \ldots$, when all three particles are on a straight line - it is called the Euler line [4] - which forms the Euler angle with horizontal $x$-coordinate (the line of symmetry $y \rightarrow-y$ ) equal to $15^{\circ}$, see Fig.1, where usually initial conditions are defined. Note that for some instance between two consecutive Euler linear configurations the positions of the bodies correspond to the vertices of an isosceles triangle with one of the bodies located on the $x$-axis. Sometimes this configuration is used to introduce initial data. Let us consider, the following ten, polynomial in coordinates and velocities, expressions numbered in italics,

(1) $L=\sum \mathbf{x}_{i} \times \mathbf{v}_{i}=0$ Angular Momentum,

(2) $E=T+V$ Total Energy (dependable)

$$
=\frac{1}{4} \log \left(\frac{3 \sqrt{3}}{2}\right) \simeq 0.23869,
$$

(3) $I_{1}=r_{12}^{2} r_{13}^{2} r_{23}^{2}=\frac{3 \sqrt{3}}{2}$,

(4) $I_{2}=3 \sum \mathbf{x}_{i}^{2}=\sum_{i<j} r_{i j}^{2}=3 \sqrt{3}$

Moment of Inertia,

(5) $T=\frac{1}{2} \sum \mathbf{v}_{i}^{2}=\frac{3}{8} \quad$ Kinetic Energy,

(6) $\tilde{T}=\mathbf{v}_{1}^{2} \mathbf{v}_{2}^{2} \mathbf{v}_{3}^{2}=\frac{1}{128}$,

$$
\begin{gathered}
J_{i}(k)=\mathbf{v}_{i}^{2}+\left(k^{2}-\frac{1}{2}\right) \mathbf{x}_{i}^{2}=\frac{1}{2}, i=1,2,3, \\
J_{s} \equiv \sum_{i=1}^{3} J_{i}\left(k_{0}\right)=2 T+\frac{1}{3}\left(k_{0}^{2}-\frac{1}{2}\right) I_{2}=\frac{3}{2} .
\end{gathered}
$$

It can be shown that all ten expressions from (1)-(10) become constants under the evolution (3), (4). Seven of them are functionally (algebraically) independent. Let us choose $L, I_{1}, I_{2}, T, \tilde{T}, J_{1}, J_{2}$. It can be shown that in spite of polynomiality of these expressions they do not generate infinite-dimensional, finitely-generated polynomial Poisson algebra with 7 generators as "seeds" as it might be expected. However, 5 of them $L, I_{1}, I_{2}, T, \tilde{T}$ do generate a commutative algebra in the following sense: all Poisson brackets being calculated on the algebraic lemniscate (1) vanish.

The Hamiltonian

$$
\mathcal{H}=T+\frac{1}{4} \ln I_{1}-\frac{\sqrt{3}}{24} I_{2},
$$

see (2), is made from particular integrals $T, I_{1}, I_{2}$. It can be shown explicitly that the Poisson bracket $\{\mathcal{H}, L\}=0$ and r.h.s. of the five Poisson brackets vanish on algebraic lemniscate (1):

$$
\{\mathcal{H}, T\}=\left\{\mathcal{H}, I_{1}\right\}=\left\{\mathcal{H}, I_{2}\right\}=\{\mathcal{H}, \tilde{T}\}=\left\{\mathcal{H}, J_{s}\right\}=0,
$$


noting that $J_{s}$ is made from $T, I_{2}$, see (10). Hence, the Hamiltonian $\mathcal{H}$ is particularly superintegrable, where $L$ is global and $T, I_{1}, I_{2}, \tilde{T}$ are four particular Liouville integrals. We were unable to find particular Liouville integrals, for which $J_{1,2,3}$ become eventually constants of motion on trajectory (11). Interestingly, it can be shown that for any positive $c$ in (3) the energy $E$ is positive and when $c$ tends to zero the energy vanishes.

We conclude that the 3-body choreographic motion with pairwise potential (2) is maximally (particularly) superintegrable: there exist 7 constants of motion. Taking velocity-independent integrals (constants of motion) (3) and (4) one can see that the 3-body choreographic motion in the $\mathbb{R}^{3}$ configuration space parametrized by squared relative distances follows a planar closed curve. The same is true in momentum space when coordinateindependent integrals (constants of motion) (5) and (6) are taken. Both curves are elliptic ones. Thus, the closed periodic trajectory in phase space is factorized: two closed periodic (elliptic) curves, one is in coordinate space and another one is in momentum space, factor out.

It has to be noted that 3-body choreographic figure8-shape motion exists for $\mathbb{R}^{2}$ Newtonian gravity, see Fig.1, at gravitational constant $G=1 / 2$, when the second term in Fujiwara et al, potential (2) vanishes, $I_{2}=0$. The figure-8-shape curve is known numerically, see [9]. This choreography is characterized by the period $T=6.904470$. The Euler line also exists in this case, its Euler angle (with $x$-coordinate) is $\sim 8.90267^{\circ}$. In general, the question about its (particular) superintegrability remains open, although total angular momentum is conserved as well as modified $\mathcal{I}_{1}$ (see below, eq.(8)).

(B) 3-body $\mathbb{R}^{3}$ Newton problem. The Hamiltonian for three point particles of unit mass is given by,

$$
\mathcal{H}=\frac{1}{2} \sum_{i=1}^{3} \mathbf{v}_{i}^{2}-\sum_{i<j}^{3} \frac{1}{r_{i j}},
$$

where the gravitational constant is assumed equal to one, $G=1$. Under special initial data the system of four coupled Newton equations in space of relative motion leads to choreographic figure-8-shape trajectory as a solution when three particles move one after another with equal time delay, see Fig.1. The Euler line exists, its Euler angle is $\sim 14.0688^{\circ}$, for details see [4]. This trajectory (and its initial data) is known numerically with high accuracy [4]. 3-body evolution can be constructed approximately, in particular, by using Mathematica code designed by Moeckel [10], unlike the case of algebraic lemniscate, where it is known in terms of Jacobi elliptic function, see (44). Using the code [10, it is found the period $T=6.325913 \ldots$ and checked that both global integrals, energy and total angular momentum, are constants of motion,

$$
E=-1.287142, L=0.000000,
$$

with accuracy of 7 figures. The next decimal digit in (77) floats being time dependable. The important guess, based on Weierstrass polynomial approximation theorem about approximation a function by polynomials, is to look for possible particular Liouville integral for (6) in a form of single argument Taylor expansion taking as entry the particular integrals of motion for algebraic lemniscate (1) in the potential (2): $I_{1}, I_{2}, T, \tilde{T}, J_{s}$, see below (1)-(10). Following this guess we manage to construct all 5 particular Liouville integrals, which Poisson-commute with the Hamiltonian (6): $\mathcal{I}_{1}, \mathcal{I}_{2}, \mathcal{T}, \tilde{\mathcal{T}}, \mathcal{J}_{s}$, see (8), (9), (10), (11), (12), on Remarkable figure-8-shape trajectory. Righthand-side of Poisson brackets is almost zero being of order of $10^{-7}$ (or less) in agreement with accuracy provided by the evolution [10], see (77). In order to check consistency it is verified these particular Liouville integrals on Remarkable figure-8-shape trajectory become constants,

$$
\begin{aligned}
\mathcal{I}_{1}= & I_{1}-0.5450972 I_{1}^{2}+0.13047597 I_{1}^{3}-0.00514404 I_{1}^{4}-0.003987607 I_{1}^{5}+ \\
& 8.130425 \times 10^{-4} I_{1}^{6}-5.441899 \times 10^{-5} I_{1}^{7}+6.4018378 \times 10^{-7} I_{1}^{8} \approx 0.7092995, \\
\mathcal{I}_{2}= & I_{2}-0.2241790 I_{2}^{2}+0.0150429 I_{2}^{3}+0.000838 I_{2}^{4}-0.0001117 I_{2}^{5}+ \\
& 0.00002001 I_{1}^{6}-4.821201 \times 10^{-6} I_{2}^{7}+3.15375 \times 10^{-7} I_{2}^{8} \approx 1.5099589, \\
\mathcal{T}_{3}= & -1.7073653 T^{2}+1.27576 T^{3}-0.0937725 T^{4}-0.53251342 T^{5}+ \\
& 0.38893304 T^{6}-0.1161555 T^{7}+0.0131440 T^{8} \approx 0.2280553, \\
\tilde{\mathcal{T}}_{3}= & \tilde{T}-11.627099 \tilde{T}^{2}+77.1466332 \tilde{T}^{3}-319.485185 \tilde{T}^{4}+845.607914 \tilde{T}^{5}- \\
& 1396.91200 \tilde{T}^{6}+1316.83205 \tilde{T}^{7}-542.334849 \tilde{T}^{8} \approx 0.03757715, \\
\mathcal{J}_{s}= & J_{s}-1.12957370 J_{s}^{2}+0.72387170 J_{s}^{3}-0.28746375 J_{s}^{4}+7.2313705 \times 10^{-2} J_{s}^{5}- \\
& 1.12265877 \times 10^{-2} J_{s}^{6}+9.801857 \times 10^{-4} J_{s}^{7}-3.66702 \times 10^{-5} J_{s}^{8} \approx 0.38492968 .
\end{aligned}
$$


Amplitudes of oscillations of the constants of motion are of the order of $10^{-7}$ or less, it implies that the last figure in (8)-(12) can be changed in one unit. Thus, the 3-body choreography with zero total angular momentum in $\mathbf{R}^{3}$ Newton gravity is maximally, particularly, superintegrable being characterized by 7 constants of motion $E, L, \mathcal{I}_{1}, \mathcal{I}_{2}, \mathcal{T}, \tilde{\mathcal{T}}, \mathcal{J}_{s}$. Hence, the 3 -body $\mathbf{R}^{3}$ Newton gravity is maximally particularly superintegrable being characterized by 6 (one global and five particular) Liouville integrals.

Conclusions. 3-body choreography with zero angular momentum exists for a number of pairwise potentials both attractive at all distances and also repulsive at small distances being attractive at large distances only [9]. We conjecture that all of them are maximally particularly superintegrable: it might be the intrinsic property of choreography explaining its existence.

It is already known that 5-body choreography on algebraic lemniscate, found in [11], is potential problem for two values of elliptic moduli in (3) with pairwise potential [12]. It is characterized by 15 explicitly-found Liouville integrals which become the constants of motion on algebraic lemniscate, hence, the trajectory is maximally, particularly superintegrable [13]. 5-body choreography on Remarkable figure-8-shape trajectory in $\mathbb{R}^{3}$ Newton gravity also exists [4]. The question about its integrability is open.

The phenomenon of choreography manifests the appearance of a new type of equilibrium configurations: moving, non-steady equilibrium.

Acknowledgements. The authors thank T Fujiwara, R Moeckel, R Montgomery and C Simó for useful mail correspondence and in some cases for personal discussions (R.M. and R.M.) and T Damour (IHES) for the important remark. A.V.T. is grateful to participants of the seminars at University of Minnesota, Simons Center for Geometry and Physics, C.N. Yang Institute for Theoretical Physics and Stony Brook University, all at Stony Brook, especially, to V Korepin and R Schrock for interest to this work. This research is partially supported by CONACyT A1-S-17364 and DGAPA IN113819 grants (Mexico).

* Electronic address: turbiner@nucleares.unam.mx
† Electronic address: vieyra@nucleares.unam.mx

[1] C. Moore,

Braids in classical dynamics, Phys Rev Lett 70, 3675 (1993)

[2] A. Chenciner, R. Montgomery, A remarkable periodic solution of the three-body problem in the case of equal masses, Ann. Math. 152, 881 (2000)

[3] A. Chenciner, J. Gerver, R. Montgomery, C. Simó, Simple Choreographic Motions of $N$ Bodies: A Preliminary Study,

In: Newton P., Holmes P., Weinstein A. (eds), Geometry, Mechanics, and Dynamics. Springer, New York, NY (2002)

[4] C. Simó,

New Families of Solutions in N-Body Problems. In: Casacuberta C., Miró- Roig R.M., Verdera J., XambóDescamps S. (eds) European Congress of Mathematics. Progress in Mathematics, vol 201. Birkhäuser, Basel (2001)

[5] T. Fujiwara, H. Fukuda, H. Ozaki, Choreographic Three Bodies on the Lemniscate, J. Phys. A36, 2791 (2003)

[6] A. Turbiner, Particular Integrability and (Quasi)-exact-solvability, Journal of Physics A46 (2013) 025203

[7] N.N. Nekhoroshev, Action-angle variables and their generalization, Trans. Moscow. Math. Soc. 26, 180-198 (1972)

[8] F. Tremblay, A.V. Turbiner, P. Winternitz, Periodic orbits for an infinite family of classical superintegrable systems, Journal of Physics A43 (2010) 015202

[9] H. Fukuda, https://kilin.clas.kitasato-u.ac.jp/3body/LJ/

[10] We thank Prof R. Moeckel (University of Minnesota) for sharing the Mathematica code for calculating the evolution; Moeckel's code is a simple interface which solves the system of coupled Newton equations with given initial conditions by using the Mathematica command NDSolveValue, following our analysis this code provides systematically a relative accuracy $\sim 10^{-7}$ leading to 7 confident significant digits (figures).

[11] T. Fujiwara, H. Fukuda and H. Ozaki, $N$-body Choreography on the Lemniscate, Developments and Applications of Dynamical Systems Theory 1369, 163-177 (2004)

[12] J.C. Lopez Vieyra, Five-body choreography on the algebraic lemniscate is a potential motion, Physics Letters A 383, 1711-1715 (2019)

[13] A.V. Turbiner, J.C. Lopez Vieyra, Modified $R^{2}$ Newtonian Gravity: Superintegrability of $(2 n+1)$-body choreographic motion on the Lemniscate, (in preparation) 